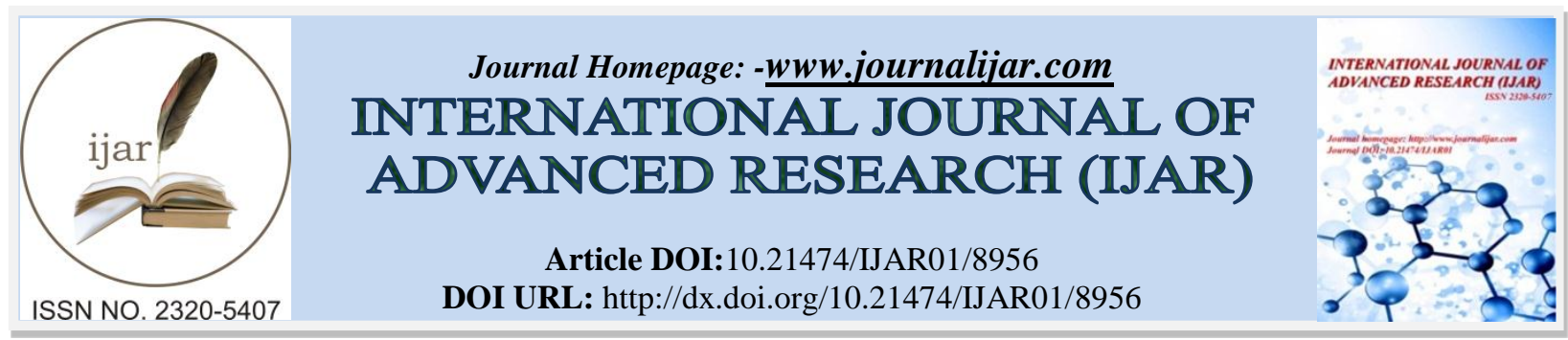

RESEARCH ARTICLE

\title{
BLOOD VESSELS SEGMENTATION FROM RETINAL IMAGES FOR DIABETIC RETINOPATHY DETECTION.
}

Manisha Laxman Jadhav ${ }^{1}$ and Dr.M. Z. Shaikh ${ }^{2}$.

1. Department of E\&TC Engineering, MET's Institute of engineering, Nashik.India.

2. Principal Bharti Vidyapeeth's College of Engineering, CBD, Belapur,Navi Mumbai,India.

\section{Manuscript Info}

Manuscript History

Received: 22 February 2019

Final Accepted: 24 March 2019

Published: April 2019

Key words:-

diabetic retinopathy, blood vessels segmentation, kirsch's template.

\section{Abstract}

In the modern world, diabetic retinopathy (DR) has become one of the most severe complication prevalent among diabetic patients. The success rate of its curability solemnly depends on the early stage diagnosis or else will lead to total blindness. Blood vessel segmentation of fundus images has obtained considerable importance during the past few years since it facilitates the early detection of eye diseases. Diabetic retinopathy (DR) is effect of diabetes mellitus to the human vision that is the major cause of blindness. Early diagnosis of DR is an important requirement in diabetes treatment. Retinal fundus image is commonly used to observe the diabetic retinopathy symptoms. It can present retinal features such as blood vessel and also capture the pathologies which may lead to DR. Blood vessel is one of retinal features which can show the retina pathologies. It can be extracted from retinal image by image processing with following stages: preprocessing, segmentation, and post-processing. This paper contains a study of public retinal image dataset and several methods for blood vessels segmentation from various conducted researches. There is no further analysis to conclude the best method which can be used for general cases. However, we suggest blood vessels segmentation using Kirsch's template method that gives the best accuracy in segmentation of blood vessels.

Copy Right, IJAR, 2019,. All rights reserved.

\section{Introduction:-}

To diagnose the diseases, the patients undergo imaging techniques. Image processing plays an important role in medical diagnosis. Examination of blood vessels has gained considerable importance during the past decades since this facilitates the early detection of diseases. The disease affecting the eyes like glaucoma and diabetic retinopathy can be analyzed by the examination of blood vessels. The fundus of the eye is the interior surface of the eye opposite the lens and includes the retina, optic disc ,macula, fovea and posterior pole. The fundus images are captured by fundus photography. Glaucoma and diabetic retinopathy are the major applications of fundus image processing. Diabetic Retinopathy (DR) is an eye disease which occurs due to diabetes. It damages the small blood vessels in the retina resulting in loss of vision. : (i) early stage or non-proliferate diabetic retinopathy, (ii) maculopathy and (iii) progressive or proliferate retinopathy. In diabetic retinopathy, fluid from the vessel leaks and gets deposited in the retinal regions. These occur in the form of yellow spots called exudates. Thus Diabetic Retinopathy can be detected 
by the presence of exudates. For effective treatment, the disease has to be detected earlier. Diabetic Retinopathy leads to damage to the blood vessels and also formation of lesions. Another complexity of the disease is that new abnormal blood vessels grow in the retina, known as revascularization. These abnormalities lead to severe visual problems. If the exudates extend to the macular region it will lead to severe vision loss. For the detection of exudates, first the blood vessels in the retinal regions are to be segmented. After the segmentation of blood vessels, optic disc is to be segmented.The blood vessels are to be removed to detect the presence of exudates.

The retinal vasculature is composed of arteries and veins appearing as elongated features, with their tributaries visible within the retinal image. There is a wide range of vessel widths ranging from one pixel to twenty pixels, depending on both the width of the vessel and the image resolution. Other structures appearing in ocular fundus images include the retina boundary, the optic disc, and pathologies in the form of cotton wool spots, bright and dark lesions and exudates as shown in Figure 1(b-d). The vessel cross-sectional intensity profiles approximate a Gaussian shape or a mixture of Gaussians in the case where a central vessel reflex is present. The orientation and grey level of a vessel does not change abruptly; they are locally linear and gradually change in intensity along their lengths. The vessels can be expected to be connected and, in the retina, form a binary treelike structure. However, the shape, size and local grey level of blood vessels can vary hugely and some background features may have similar attributes to vessels as illustrated in Figure 1(a and d). Vessel crossing and branching can further complicate the profile model. As with the processing of most medical images, signal noise, drift in image intensity and lack of image contrast pose significant challenges to the extraction of blood vessels. Retinal vessels also show an evidence of a strong reflection along their centerline known as a central vessel reflex as evident in Figure 1(a), which is more apparent in arteries than veins, is stronger at images taken at longer wavelengths, and typically found in the retinal images of younger patients.

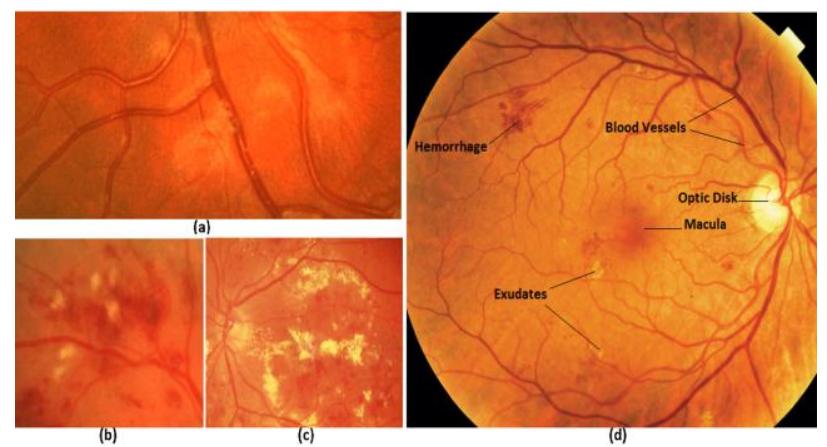

Figure 1:-Morphology of retinal images:

(a) central vessel reflex and uneven background, (b) cotton wool spots, (c) hard exudates, (d) anatomical structures in the retina.

Retinal vessel segmentation algorithms are a fundamental component of automatic retinal disease screening systems. The paper examines the blood vessel segmentation methodologies in two dimensional retinal images acquired from a fundus camera and a survey of techniques is presented. The aim of this paper is to study, analyze and categorize the retinal vessel extraction algorithms, techniques and methodologies, giving a brief description, highlighting the key points and the performance measures.

In this paper the performance of blood vessel segmentation is evaluated using following methods

1. Retinal Blood Vessel Segmentation Using Global Threshold

2. Characterization of Changes in Blood Vessel for Segmentation

3. Blood Vessel Segmentation Using Spatial Filtering With Krisch's Template

4. Blood Vessel Segmentation Using Edge Detection

The performance is evaluated using PSNR, RMSE and Correlation of coefficient

This paper is organized as follows: Section 2 presents the preprocessing for image enhancement and noise removal. Section 3 explains the algorithm used for optic disc elimination. Section 4 explains in details the method for detection of microaneurysms and hemorrhages detection presented in this paper; Section 5 explains the various features extraction for machine learning algorithm. Section 6 deals with the classification algorithms such as SVM and neural 
network. Section 7 shows the results achieved by the proposed method and, finally, Section 8 presents the conclusion of this article.

\section{Retinal Blood Vessel Segmentation Using Global Threshold Method}

The morphology of blood vessels in retinal fundus images is an important indicator of diseases like glaucoma, hypertension and diabetic retinopathy. The accuracy of retinal blood vessels segmentation affects the quality of retinal image analysis which is used in diagnosis methods in modern ophthalmology. Contrast enhancement is one of the crucial steps in any of retinal blood vessel segmentation approaches. The reliability of the segmentation depends on the consistency of the contrast over the image. This section presents an assessment of the suitability of a recently invented spatially adaptive contrast enhancement technique for enhancing retinal fundus images for blood vessel segmentation. The enhancement technique was integrated with a variant of Tyler Coye algorithm, which has been improved with Hough line transformation based vessel reconstruction method [1].

\section{Tyler Coye Algorithm}

Figure 2 shows the flow chart of the Tyler Coye algorithm for blood vessel segmentation in retinal images.

1. The principle component analysis (PCA) of weighted Lab color model is used for converting the image into grayscale.

2. The contrast enhancement is done by adaptive histogram equalization resulting into exclusion of the background by subtracting the average filtered image.

3. Isodata[2] is used for extracting a fair threshold level for the binarization process and finally, the smaller components are removed by considering the size of each connected components.

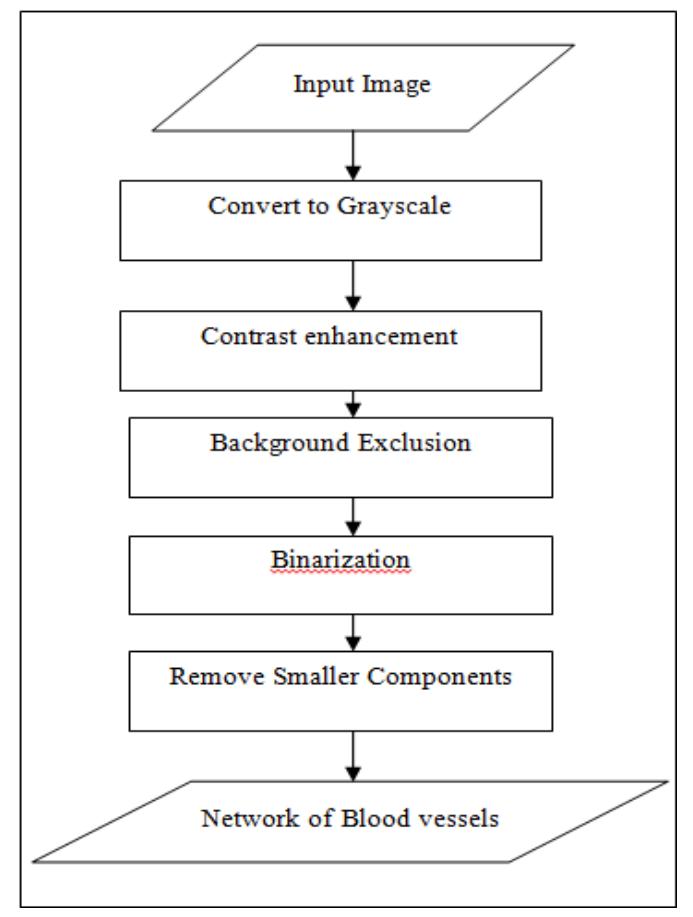

Figure 2:-Flow of Tyler Coye Algorithm

\section{Integration of SUACE}

The SUACE algorithm is injected into the process at the contrast enhancement step. The input for the SUACE is the gray scale image which is obtained by PCA based Lab color to grayscale conversion. It is common that any contrast enhancement algorithm amplifies both the signal and noises simultaneously. In this application, the amplified noises appeared as several connected components which are detached from the network of blood vessels. The simple connected component analysis method which is used in the original implementation of Tyler Coye tends to remove parts of some smaller vessels with the width of a single pixel, due to the discontinuities over the vessel network. This original noise removal technique is extended by fixing the discontinuities in the vessel network as follows: 
1. Remove the connected components with the area less than a1. The value of a1 is selected to be a smaller value somewhere between 5-15 pixels.

2. Obtain the probabilistic Hough line transformation of every local window with the size $\mathrm{h}$ and fill the disconnected gaps in the vessel network by drawing lines for the each pair of endpoints having the line votes more than the given threshold $\mathrm{v}$.

3. Remove the connected components with the area less than a 2 where a2>>a1.

The selected a1, as it does not remove any of the vessels in the image, the first step can remove some of the noises safely. The window size $\mathrm{h}$ was selected based on the average distance of the discontinued spaces in the vessel networks. Since the window is small, the threshold for the Hough line votes should be kept smaller. The value of $\mathrm{v}$ is empirically selected throughout the experiments. After fixing the discontinuities, only the noises are remained without connecting to the vessel network. Therefore a larger value to a2 can be set in the third step to remove all the noises safely without false classifying the vessels as the background. The Hough line transformation votes for potential lines having similar angles hence a slight discontinuity in a vessel does not affect the voting process. Therefore the discontinuities can be fixed computationally by drawing lines with the highest votes

\section{Retinal Blood Vessel Segmentation Using Characterization of Changes in Blood vessel}

This method discusses a technique for measuring vessel width and tortuosity in the posterior pole of the retina, and shows how it can be used to demonstrate statistically significant changes in these parameters for ROP subjects. The technique firstly implements segmentation of the retinal vasculature. This is carried out using morphological preprocessing based on linear structuring elements, followed by enhancement using a smoothed second-derivative operator. A final stage of morphological post-processing is then used, prior to thresholding. This results in a binary mask representing detected vessels from which vessel widths can be calculated. This binary mask is then skeletonised. The tortuosity of segments of this skeleton (corresponding to specific blood vessels) can be calculated. By considering the average vessel width and tortuosity at the posterior pole, it can be shown that

1. subjects with threshold disease have wider and more tortuous vessels, though only the changes in vessel width can be considered to be statistically significant based on our measurement technique,

2. that subjects who progress to threshold disease subsequent to their first examination experience a statistically significant increase in average vessel width

3. that treatment leads to a rapid reduction in average vessel width and tortuosity.[3]

The work presented here focuses on quantifying the thickness and tortuosity of the retinal vasculature being screened for sight-threatening ROP.

1. A first stage is to segment the vasculature from other anatomical structures in the image such as the optic disk, by creating a binary mask image of the complete vasculature.

2. This output mask image marks pixels in the original picture that are part of vessels as white (binary 1) with all other pixels as black (binary 0). Accurate vasculature segmentation is fundamentally important, as further analysis of vessel properties depends on the accuracy of this segmentation.

3. In developing this algorithm, two types of retinal images are considered-black and white images obtained using fluorescein angiography, and colour images obtained using a wide-angle retinal camera under white light.

4. In fluorescein angiography, a contrast enhancement dye (fluorescein) is injected into the blood stream, which fluoresces under ultraviolet light. Fluorescein angiograms typically have higher contrast than colour fundus images, and segmentation of vessels in fluorescein angiograms using our system is more successful than in the colour images.

The method presented in [3] uses a morphological filter to emphasise linear structures such as vessels. The differential properties of the vessels provide additional emphasis. An additional morphological filter, and hysteresis thresholding is used to obtain the final binary image which represents the mask of the vessels.

Morphological image processing is particularly good at extracting image features whose shape is known a priori. In particular, morphology provides an attractive solution for detecting quasi-linear shapes such as vessels in an image. Since the vasculature is known to be piecewise linear, an algorithm that robustly extracts linear shapes is useful in vasculature extraction.

The aim of the initial morphological filtering operation is to emphasise the vasculature, preserving vessel crossings and bifurcations. The approach used in this project uses the difference between the supremum and infinumb of the openings of the original image $\mathrm{I} 0(\mathrm{x}, \mathrm{y})$ with two linear structuring elements of different lengths. 


\section{Retinal Blood Vessel Segmentation Using Spatial Filtering with Krisch's Template}

In [4] a new algorithm to detect the blood vessels is explained. The initial enhancement of the image is carried out using Adaptive Histogram Equalization, followed by this the curvelet Transforms are applied to the equalized image and the curvelet coefficients are obtained. The modifications to the Curvelet transform coefficients are carried out by suppressing all the coefficients of one band. The combined effect of the equalization and the Curvelet Transforms provides a better enhancement to the image. This enhanced image is used for the extraction of the blood vessels. The vessel extraction is done based on thresholding technique and the Kirsch's templates. It involves spatial filtering of the image using the templates in eight different orientations. The masking of redundant regions in the obtained output image is carried out using boundary techniques. The salt and pepper noise in the image are removed by applying median filtering to the image.

\section{Retinal Image Enhancement using Adaptive Histogram Equalization}

Since the adaptive histogram equalization is capable of improving the image's Local Contrast in bringing out more details of the image, it acts as a good tool for the enhancement of the edges. Comparison of the input and the enhanced image is shown in figure 3 .

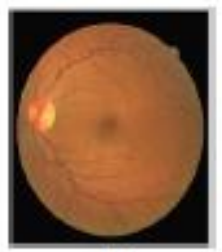

(a)

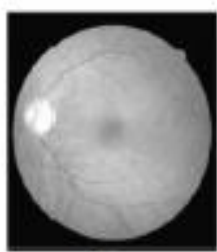

(b)

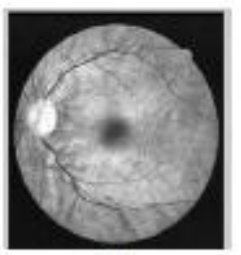

(c)

Figure 3:-Image Enhancement Using Adaptive Histogram Equalization (a) Original Image (b) Gray Scale image (c) Adaptive Histogram Equalized image

\section{Retinal Image Analysis Using Curvelet Transform (FDCT)}

The Curvelet Transform is well adapted to represent the edges; it is good candidate for analysis of edges. The curvelet coefficients can be modified to further enhance the image contrast.

Consequently this method to analyze the retinal image consists of the following steps:

Applying FDCT via wrapping method results into a set of scales, $\mathrm{Sj}$; each scale consists of a set of directional bands Di containing coefficients.

For the directional band, $\mathrm{D}\{1\}\{1\}$; do the following:

1. Calculate the minimum value.

2. Replace all the coefficients with the minimum value

Reconstruct the enhanced image using modified curvelet coefficients.

\section{Image fusion}

The results obtained in section $\mathrm{C}$ are compared with results of section $\mathrm{B}$ based on the following algorithm:

1. Find the pixel value of the enhanced (a1) and reconstructed image (a2).

2. Fix the threshold value.

3. Compare each pixel of both the images and do the following:

1. If a1 and 2 are less than threshold, or greater than threshold, find Mean value and replace

2. If $\mathrm{a} 1$ is less than threshold and $\mathrm{a} 2$ is greater than threshold, replace with a2.

3. If a2 is less than threshold and a1 is greater than threshold, replace with a1.

\section{Extraction of Blood vessels using Kirsch's Templates}

The extraction of blood vessels from the enhanced image is done based on the Kirsch templates. The method involves spatial filtering using the templates of different orientations followed by thresholding technique. Variations in the output image can be obtained by changing the value of threshold used. The maskings of redundant areas of the result are carried out using boundary technique. 


\section{Smoothing of Image using Median Filtering}

To the results obtained in stage $\mathrm{F}$, for the purpose of removal of the noisy areas in the image, Median filtering is applied and the image is smoothened.

\section{Retinal Blood Vessel Segmentation Using Edge Detection}

Edge detection is the most common approach for detecting discontinuities in an image. It is considered as the boundary between two regions having distinct intensity levels or having distinct gray level. Edge is a set of connected pixels that lie on the boundary between two regions. Edge detection locates sharp changes in the intensity function. Edges are pixels where brightness changes abruptly. There are many edge detectors such as Robert, Priwitt, Sobel, Canny etc. In [5] edge detection is done using morphological approach. Simple morphological operators are used for segmentation to remove optic disc.

Figure 4 shows the proposed diagram of blood vessel segmentation using edge detection.

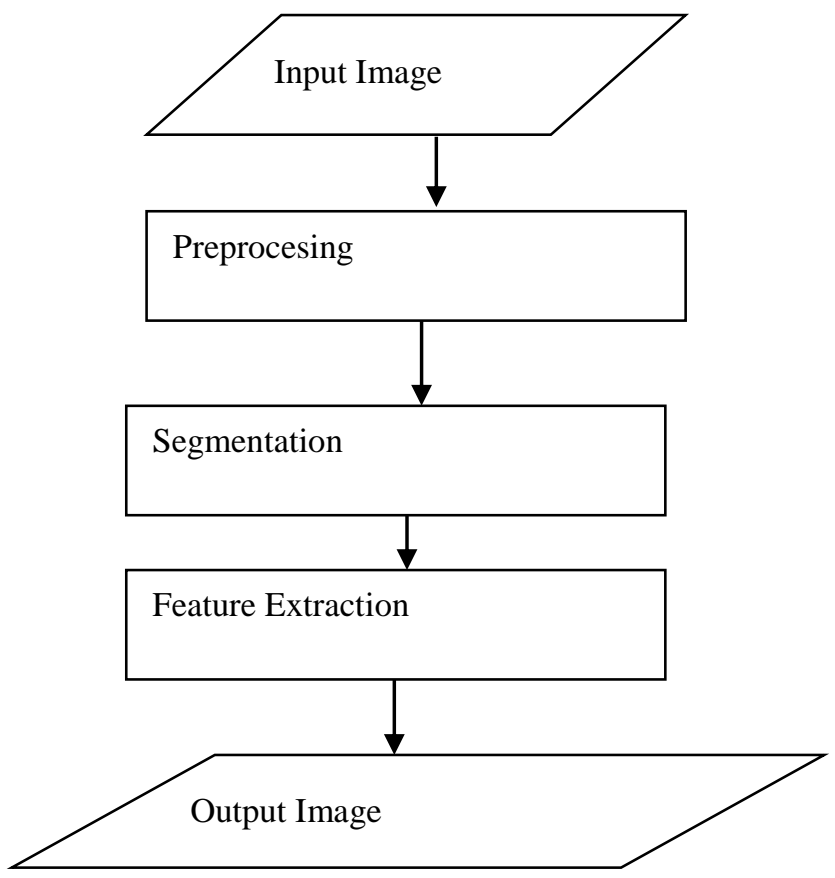

Figure 4:-Blood Vessel Segmentation Using Edge Detection

\section{Pre-Processing Methodology:-}

Input image is pre-processed to achieve enhanced image.

1. Extract the RGB components of the input image

2. Image after RGB extraction is used for histogram equalization. Instead of normal histogram equalization the present work use adaptive histogram equalization.

3. Then wavelet denoising is used to remove the noise.

Adaptive histogram equalization is used for image enhancement. Histogram equalization automatically determines a transformation function that seeks to produce an output image that has a uniform histogram. When automatic enhancement is desired, this is good approach, because the result from this technique is predictable and the method is simple to implement. Wavelet denoising is employed for noise removal.

The preprocessed image is highly enhanced denoised image which improves image quality to proceed for image segmentation. 
Figure 5:-a) shows the original image and figure $5 \mathrm{~b}$ shows the pre-processed image.

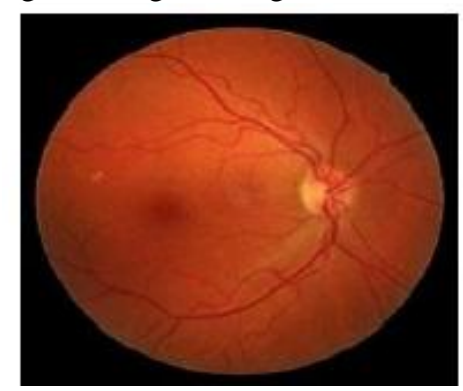

(a)

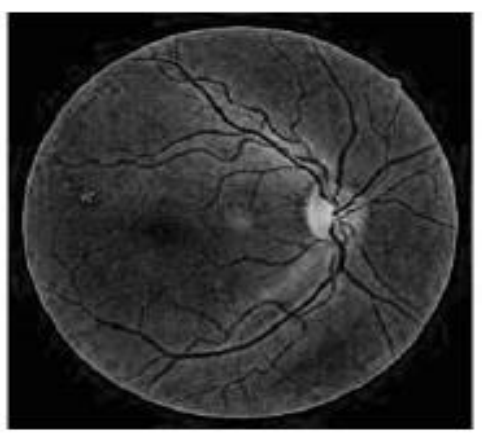

(b)

Figure 5:-Retinal Image Pre-processing (a) Original Image (b) pre-processed Image

\section{Image Segmentation}

Image segmentation is the process of partitioning a digital image into multiple number of segments or sets of pixels. The postcode is extracted from the part of image. Segmentation subdivides an image into its various regions or objects. Image segmentation is done using various morphological operations [6].

\section{Morphological operations}

Principle logic operation used in image processing are AND, OR and NOT. NOT is the complimentary operation. Logic operations such as AND, OR are performed on a pixel by pixel basis between corresponding pixels of two or more images. NOR operation is performed on the pixel of single image. Complimentary operation converts the black pixel into white and the white pixel into black. First the complimentary image of the pre-processed image is taken. Then it is taken for dilation, erosion operation. Dilation and erosion are fundamental morphological operators. One of the simplest application of dilation is bridging gaps. During dilation the pixels are added to the region and during erosion the pixels are removed from boundary. Dilation expands an image and erosion shrinks it. In this method the present work use opening operator. Opening is erosion followed by dilation. Closing is dilation followed by erosion. Opening generally smoothens the contours of an image. Opening and closing operations may be considered as "second level" operations; in that they build on the basic operations of dilation and erosion. In opening operation, each case the image has been separated into distinct components, and the lower part has been removed completely. With closing, the image will be fully joined up. Thus a joining-up effect is obtained with the image, using a diagonal structuring element.

\section{Optic disc removal}

Optic disc removal is a major task during retinal blood vessel segmentation. Several methods have been put forward for optic disc localization [7]. The optic disc is removed by subtracting the image after opening from adaptive histogram equalization.

After optic disc removal the image is adjusted to increase its contrast. After adjusting operation the blood vessel is observed brighter than the previous image. 
Figure 6:-shows the optic disc removed image.

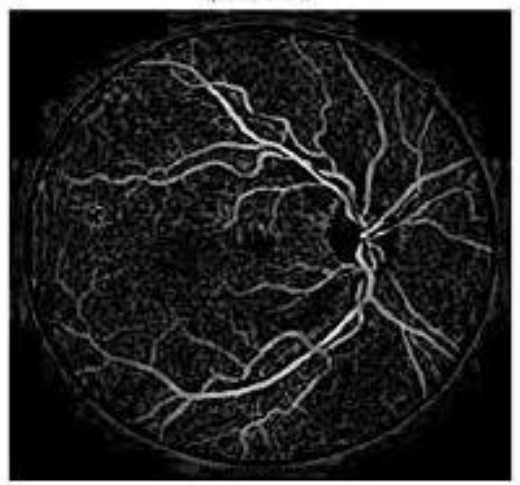

\section{Binarization}

This image is converted into binary format. Binary image have only two values: 0 and 1 . If background and object pixels have gray levels grouped into 2 dominant modes, they can be separated with a threshold. The gray scale image is converted into black and white image after binarisation. Lighter region is converted to white and the darker area to black pixel.

In the final step, the image with extracted blood vessels is resulted. From a binary image all connected components (objects) are removed. Connected components that have fewer than P pixels produce another binary image, BW2. This operation is known as an area opening operation used to obtain the final image.

Figure 7:-shows the blood vessels extracted from the image.

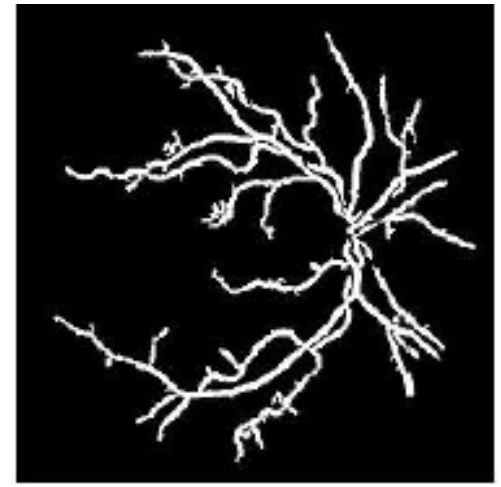

\section{Results and Performance Evaluation:-}

DRIVE dataset is used for measuring the performance of blood vessel segmentation algorithms. The dataset provides ground truth computer generated blood vessel segmentations which can be used as gold standard for performance evaluation. The performance is evaluated using PSNR, RMSE and Correlation of coefficient

Figure 8:-shows the performance evaluation of various blood vessel segmentation techniques for the sample image from DRIVE dataset.

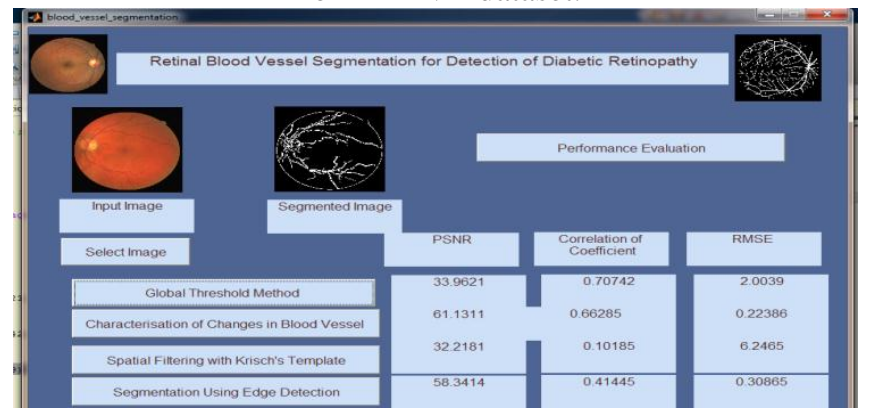


The performance of various image blood vessel segmentation techniques is depicted in table 1

\begin{tabular}{|l|l|l|l|}
\hline Blood Vessel Segmentation Algorithm & PSNR & $\begin{array}{l}\text { Correlation of } \\
\text { Coefficient }\end{array}$ & RMSE \\
\hline Global Threshold & 34.7061 & 0.0634 & 3.8285 \\
\hline Characterization of Changes in Blood Vessel & 57.1343 & 0.0693 & 0.3565 \\
\hline Spatial Filtering with Krisch's Template & $\mathbf{3 0 . 9 5 8 3}$ & $\mathbf{- 0 . 0 0 2 8}$ & $\mathbf{7 . 3 8 8 9}$ \\
\hline Edge Detection & 56.737 & 0.0438 & 0.3726 \\
\hline
\end{tabular}

Table 1:-Performance Evaluation of Various Blood Vessel Segmentation Techniques

Figure 9 shows the performance evaluation of various blood vessel segmentation algorithms in terms of PSNR for DRIVE Dataset (40 Instances). The lower value of PSNR and Correlation Coefficient and the higher value of RMSE proves the proposed fusion method to be the best amongst all the compared methods.

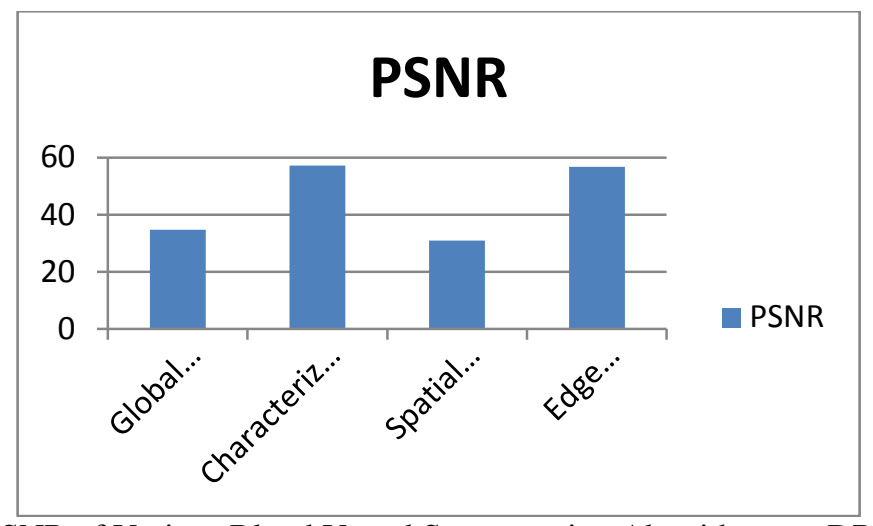

Figure 9:-PSNR of Various Blood Vessel Segmentation Algorithms on DRIVE Dataset

Figure 10 shows the performance evaluation of various blood vessel segmentation algorithms in terms of Correlation of Coefficient for DRIVE Dataset (40 Instances)

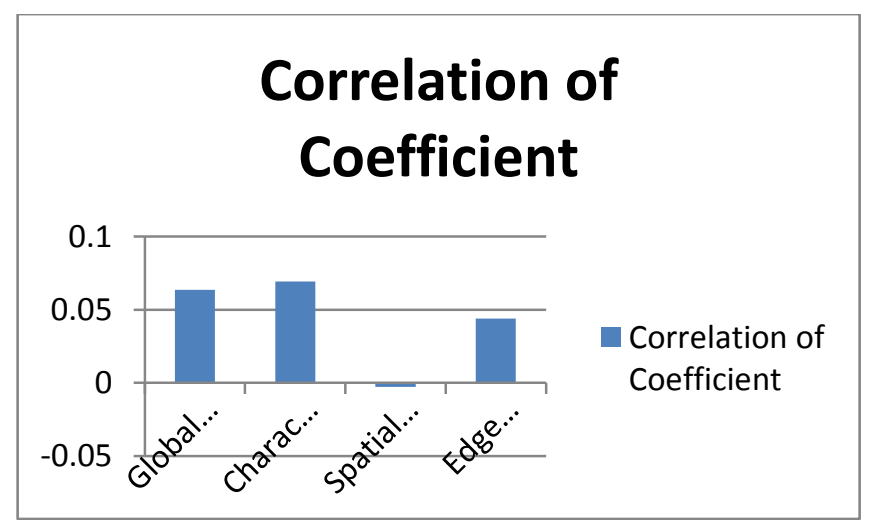

Figure 10:-Correlation of Coefficient of Various Blood Vessel Segmentation Algorithms on DRIVE Dataset

Figure 11 shows the performance evaluation of various blood vessel segmentation algorithms in terms of RMSE for DRIVE Dataset (40 Instances) 


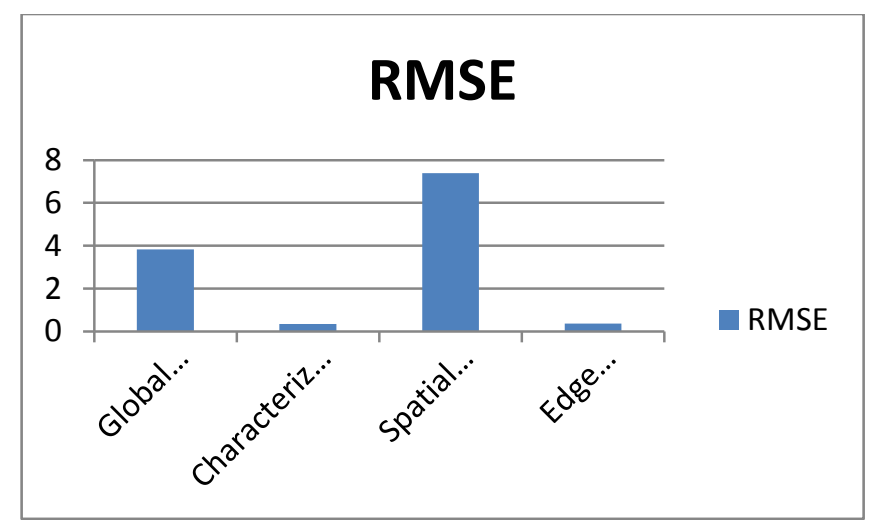

Figure 11:-RMSE of Various Blood Vessel Segmentation Algorithms on DRIVE Dataset

\section{Conclusion:-}

An automated blood vessel segmentation method can be integrated into a pre-screening system for early detection of diabetic retinopathy. Blood vessel segmentation involves a huge challenge as images present inadequate contrast, lighting variations, noise influence and anatomic variability, affecting retinal background texture and the blood vessels structure. In this paper the performance of blood vessel segmentation is evaluated using following methods

1. Retinal Blood Vessel Segmentation Using Global Threshold

2. Characterization of Changes in Blood Vessel for Segmentation

3. Blood Vessel Segmentation Using Spatial Filtering With Krisch's Template

4. Blood Vessel Segmentation Using Edge Detection

The performance is evaluated using PSNR, RMSE and Correlation of coefficient. The lower value of PSNR and Correlation of coefficient and the higher value of RMSE proves the Blood Vessel Segmentation Using Spatial Filtering With Krisch's Template to be the best for blood vessels segmentation.

\section{Acknowledgment:-}

The authors would like to thank HRF database center, DRIMDB database center, Navkar Hospital nashik, Sahyadri Hospital Pune, for their support in providing the retinal images.

\section{References:-}

1. A. M. R. R. Bandara and P. W. G. R. M. P. B. Giragama, "A retinal image enhancement technique for blood vessel segmentation algorithm," 2017 IEEE International Conference on Industrial and Information Systems (ICIIS), Peradeniya, Sri Lanka, 2017, pp. 1-5. doi: 10.1109/ICIINFS.2017.8300426

2. A. El-Zaart, "Images thresholding using ISODATA technique with gamma distribution," Pattern Recognit. Image Anal., vol. 20, no. 1, pp. 29-41, 2010.

3. Conor Heneghan, John Flynn, Michael O'Keefe, Mark Cahill, "Characterization of changes in blood vessel width and tortuosity in retinopathy of prematurity using image analysis", Elsevier,Medical Image Analysis 6 (2002) 407-429

4. M. Kalaivani, M. S. Jeyalakshmi, Aparna.V, “ Extraction Of Retinal Blood Vessels Using Curvelet Transform And Kirsch's Templates", International Journal of Emerging Technology and Advanced Engineering,ISSN 2250-2459, Volume 2, Issue 11, November 2012.

5. Surya G, Pratheesh M Vincent, Shanida K, "Automatic method for retinal blood vessel segmentation", International Journal of Recent Advances in Engineering \& Technology (IJRAET) ISSN (Online): 2347 - 2812, Volume-4, Issue -7, 2016

6. R. M. Haralick and L. G. Shapiro, "Image segmentation techniques", CVGIP, 29, pp. 100-132, 1985.

7. Deepali A. Godse, Dr. Dattatraya S. Bormane "Automated Localization of Optic Disc in Retinal Images", (IJACSA) International Journal of Advanced Computer Science and Applications, Vol. 4, No. 2, 2013. 